\title{
Algae in abundance: au natural, or a violation of Xenopus husbandry standards?
}

\author{
X \\ enopus laevis, the African Clawed \\ Frog, is well known to many \\ laboratory animal facilities. This \\ extremely aquatic and unique animal has \\ no tongue, no externally visible ears, claws \\ on some of its toes, a lateral line system \\ analogous to that seen in fish, and in the \\ wild is found in warm, stagnant ponds that \\ are typically covered with algae. It is this last \\ characteristic that became problematic for \\ the Great Eastern University IACUC. \\ Dr. Simon Eaton had three 25-gallon \\ tanks, each with two female $X$. laevis \\ frogs which he kept in an $80^{\circ} \mathrm{F}$, \\ temperature-controlled room near his \\ office. Because Eaton was the only Xenopus \\ user on campus, and because the number \\ of frogs was small, the IACUC allowed
}

Eaton to care for his own animals. The lab staff fed the animals daily and manually changed $10 \%$ of the dechlorinated water weekly. If any problems were noticed, the lab staff would immediately contact the vivarium. The vivarium's animal care and veterinary staff checked the frogs twice weekly.

During a routine semi-annual inspection by the IACUC, the inspectors reported that they could barely observe the frogs due to algae overgrowth in the tanks. This was cited as a potential significant violation of husbandry standards. When questioned, Eaton explained that his new technician wanted to keep the tanks as natural as possible for the frogs, and because they normally lived in stagnant, algae covered ponds, he agreed to letting the tanks become covered with algae. Eaton added that he had initially discussed this with the animal facility personnel, and they accepted his rationale. There were no health problems with the animals, so he did not see why the IACUC should be concerned.

Should the IACUC be concerned or was Eaton's lab acting within the standards of care for X. laevis?

\section{Jerald Silverman $\bowtie$ \\ University of Massachusetts Medical School, \\ Worcester, MA, USA.

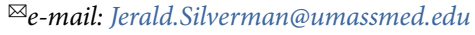

Published online: 23 July 2020

https://doi.org/10.1038/s41684-020-0595-0

\section{The clarity of SOPs}

W hile The Guide states that "the success and adequacy of the [aquatic] system depend on its ability to match the laboratory habitat to the natural history of the species ${ }^{\text {"1 }}$, the system also needs to be conducive to the specific research activities being performed. In Dr. Eaton's case, the presence/absence of algae became a research variable.

The specificity required for aquatic husbandry standards is significantly less than what is available for other species; specifically: "The type of life support system used depends on several factors including the natural habitat of the species, age/size of the species, number of animals maintained, availability and characteristics of the water required, and the type of research ${ }^{1}$." This does not, however, imply that research-driven husbandry practices for aquatics should always be described in an IACUC-approved protocol.

Great Eastern University (GEU)'s IACUC established a mechanism to review and approve "PI-husbandry" for these animals. It was at this time that Eaton and the Attending Veterinarian (AV) should have established standard operation procedures (SOPs) for husbandry and AV management of the animals and the room. These SOPs would specify, at an institutional level, the criteria for evaluating and maintaining, for example, water quality, animal health, and sanitization. Any modifications to the SOPs should be reviewed and approved by the AV prior to implementation. Furthermore, any deviations from the institutionally accepted husbandry standards would need to be detailed and justified in the IACUC-approved protocol.

The concern for Eaton and GEU arose when IACUC members became concerned that the health and welfare of the animals were jeopardized due to assumed poor husbandry practices. Adequate visualization of animals is a general necessity; however, there are other ways IACUCs can determine that there are satisfactory husbandry practices and animal health oversight in place. For example, along with the established husbandry SOPs, Eaton, his lab members, and veterinary staff perform biweekly inspections, and must maintain room sheets detailing variables such as animal health checks, water quality assessments, and room temperature. In addition, viewing animal health (or treatment) reports for Eaton's animals would show how frequently (or not) the veterinary staff identified any health or welfare concerns.

The remaining question is whether Eaton consulted with the AV before making the change in husbandry practices and, if not, would the AV have considered this a departure from the institutionally accepted husbandry standards, requiring IACUC review. GEU should have a well-described process for oversight of "PI-husbandry" areas, which includes, for example, the extent of veterinary oversight, SOPs for the care of the animals, and clear criteria for when modifications require veterinary and/ or IACUC review and approval. Since it appears that the health and welfare of Eaton's animals is not in question, GEUs IACUC should be concerned if a formalized process for oversight of "PI-husbandry" areas has not been established, if the IACUC is not aware of it, and/or if the PI has not been properly educated and/or informed of the requirements as a "PI-husbandry" lab.

\section{Lauren Danridge $\mathrm{M}^{\varpi}$}

University of Michigan Animal Care \& Use Office

(ACUO), Ann Arbor, MI, USA.

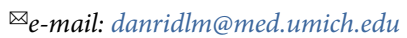

Published online: 23 July 2020

https://doi.org/10.1038/s41684-020-0596-Z

\footnotetext{
References

1. Institute for Laboratory Animal Research. Guide for the Care and Use of Laboratory Animals 8th ed. (National Academies Press, Washington, DC, 2011).
} 